\title{
Commentaries
}

\section{A key cytokine unlocks the door}

The synthesis and secretion of a variety of pro-inflammatory cytokines are increased in the chronic inflammatory reaction associated with Helicobacter pylori infection. ${ }^{1}$ In this issue (see page 24), Shibata and colleagues confirm increased secretion of tumour necrosis factor (TNF) by the inflamed mucosa, and provide evidence that during $H$ pylori infection there is both secretion of TNF and of the plasma membrane receptors for TNF, which are cleaved and secreted by gastric epithelial cells. Further experiments suggest that this secretion of soluble TNF receptors may be important in allowing the epithelial cell to protect itself from TNF induced death. Although there are relatively few normal controls in the human biopsy study, these results do suggest that the secretion of soluble TNF receptors may be an important defensive reaction, and may have implications for other inflammatory conditions of the gastrointestinal tract.

Since its discovery over 20 years ago, TNF has been recognised as an important mediator in the pathogenesis of a wide variety of diseases. TNF is one of family of related peptides, including Fas ligand and nerve growth factor, that acts through specific related cell surface associated receptors. $^{2}$ The multiple biological effects of TNF are mediated through two specific, high affinity cell surface receptors (a $55 \mathrm{kDa}$ TNF-R1 and a $75 \mathrm{kDa}$ TNF-R2) which are found on most mammalian cells. Both TNF receptors may exist in soluble forms by proteolytic cleavage of the extracellular domain of the TNF receptor or by the translation of specific transcripts formed by alternative splicing mechanisms. Enzymes responsible for the shedding of TNF and for its receptors are currently being investigated, as are the roles of "decoy", non-functioning receptors. As in all highly regulated systems, mechanisms exist to regulate the effects of important mediators and to this effect, soluble TNF receptors seem to adjust TNF bioactivity in a dose dependent manner. At high concentrations they compete for TNF binding and reduce TNF bioactivity $^{3}$ and at lower concentrations they stabilise the trimeric structure of TNF, thereby providing a reservoir for the slow release of TNF bound to its receptor. ${ }^{4}$ Measuring increased plasma concentrations of soluble TNF receptors has been proposed for the diagnosis of several neoplastic and inflammatory diseases. ${ }^{5}$

Although only one of many cytokines in a complex soup, TNF has a variety of biological effects which may be relevant to the understanding of the pathogenesis of diseases associated with $H$ pylori. These include chronic incitement of the inflammatory cascade, immunomodulation, cytotoxicity (including apoptosis), and even mitogenic stimulation and/or tumour promotion. ${ }^{2}{ }^{6}$ By examining the secretion of soluble TNF receptors in $H$ pylori associated, TNF induced apoptosis, Shibata and colleagues' paper adds to our understanding of the complex regulatory systems that must be in place to limit TNF induced damage. However, many other issues related to the interaction between TNF and $H$ pylori have still to be resolved. For example, it is not clear how TNF-R1 is somehow capable of transmitting several, sometimes antagonistic, signals. The intracellular portion of TNF-R1 contains a cytoplasmic death domain that is required for the signalling of certain activities such as apoptosis and activation of transcription factors nuclear factor $\kappa \mathrm{B}(\mathrm{NF}-\kappa \mathrm{B})$ and $\mathrm{AP}-1$. Binding of TNF to the extracellular domain of TNF-R1 induces receptor trimerisation, allowing the death domain of TNF-R1 to recruit the adapter proteins TRADD (TNF receptor associated death domain), FADD (Fas associated death domain), TRAF2 (TNF receptor associated factor), and RIP (receptor interacting protein). ${ }^{7}$ This TNF-R1 complex then activates signalling cascades leading to apoptosis, activation of JNK/SAPK (jun kinase/stress activated protein kinase) pathways and NF- $\mathrm{NB}$ activation. Although FADD interacts directly with the apoptotic proteases, thus triggering cell death, stimulation of cytoprotective gene expression mainly occurs through specific TRAFs. Experiments using $\mathrm{TRAF}^{-/-}$mice have suggested that transcription of cytoprotective genes by TRAF2 is the first and the most important role of TNF gene stimulation as these mice were unable to upregulate JNK/SAPK activity after TNF treatment, yet were highly sensitive to TNF dependent apoptosis. ${ }^{8}$ TNF-R1 mediated cell survival also seems to involve RIP, dominant negative forms of which can inhibit $\mathrm{NF}-\kappa \mathrm{B}$ and JNK activation, and overexpression of which induces both JNK and NF- $\mathrm{NB}$. During infection with $H$ pylori, does TNF act predominantly to damage epithelial cells, or to protect them from injury? In cell culture experiments, there is evidence that TNF can augment $H$ pylori induced apoptosis, ${ }^{9}$ as well as data showing that $H$ pylori can induce NF- $\mathrm{NB} .{ }^{10}$ Cell lines derived from gastric cancer may not be the best models for examining the effects of TNF or of $H$ pylori however. This is because despite TNFs original designation as an agent causing tumour cell death, most cancer cells are usually resistant to apoptosis induced by TNF unless sensitised by translational inhibitors or blockers of the TRAF2/NF- $\mathrm{BB}$ pathway. Infecting mice lacking the $55 \mathrm{kDa}$ TNF receptor with $H$ pylori could clarify the role of TNF in $H$ pylori induced gastritis and apoptosis. These mice, which are moderately resistant to the lethal effects of lipopolysaccharide but highly susceptible to infection by Listeria monocytogenes, ${ }^{11}$ may teach us whether TNF is more important for inducing the inflammatory process or in protecting the mucosa from damage.

The pharmacological modulation of TNF in chronic inflammatory diseases such as rheumatoid arthritis and Crohn's disease is already showing great promise, but our knowledge of how the gut responds to and regulates TNF is still at a relatively primitive stage. The observation that TNF may provide mitogenic signals to cancer cells may be surprising, ${ }^{6}$ especially as TNF was first described as a substance capable of killing cancer cells, but it serves to illustrate how difficult it can be to predict the biological effects of the same substance in different systems. In this context, we should not be surprised if further paradoxical effects of TNF are observed as the mechanisms of its regulation are uncovered.

H SHIRIN S F MOSS

Department of Medicine,

Division of Gastroenterology,

St Luke's-Roosevelt Hospital Centre,

College of Physicians and Surgeons,

Columbia University, New York, NY 10025, USA

Correspondence to: Professor Moss (email: smoss1@pol.net). 
1 Bodger K, Crabtree JE. Helicobacter pylori and gastric inflammation. $\mathrm{Br}$ Med Bull 1998;54:139-50.

Med Bull 1998;54:139-50. families. N Engl f Med 1996;334:1717-25.

3 Ashkenazi A, Marsters SA, Capon DJ, et al. Protection against endotoxic shock by a tumor necrosis factor receptor immunoadhesin. Proc Natl Acad Sci USA 1991;88:10535-9.

4 Aderka D, Engelmann H, Maor Y, et al. Stabilization of the bioactivity of tumor necrosis factor by its soluble receptors. F Exp Med 1992;175:323-9.

5 Aderka D. The potential biological and clinical significance of the soluble tumor necrosis factor receptors. Cytokine Growth Factor Rev 1996;7:231-40.

6 Liu RY, Fan C, Mitchell S, et al. The role of type I and type II tumor necrosis factor (TNF) receptors in the ability of TNF- $\alpha$ to transduce a proliferative signal in the human megakaryoblastic leukemic cell line Mo7e. Cancer Res 1998;58:2217-23.
7 Ashkenazi A, Dixit VM. Death receptors: signaling and modulation. Science 1998;281:1305-8.

8 Yeh WC, Shahinian A, Speiser D, et al. Early lethality, functional NF-кB activation, and increased sensitivity to TNF-induced cell death in TRAF2deficient mice. Immunity 1997;7:715-25.

9 Wagner S, Beil W, Westermann J, et al. Regulation of gastric epithelial cell growth by Helicobacter pylori: evidence for a major role of apoptosis. Gastroenterology 1997;113:1836-47.

10 Keates S, Hitti YS, Upton M, et al. Helicobacter pylori infection activates NF-кB in gastric epithelial cells. Gastroenterology 1997;113: 1099-109.

11 Pfeffer K, Matsuyama T, Kundig TM, et al. Mice deficient for the $55 \mathrm{Kd}$ tumor necrosis factor receptor are resistant to endotoxic shock, yet succumb to Listeria monocytogenes. Cell 1994;78:681-92.

\section{Glutamine in parenteral nutrition: more food for thought}

Until recently glutamine had not been included in commercial parenteral nutrition solutions. This is because of concerns about its spontaneous degradation, which results in the formation of pyroglutamic acid and ammonia. However, the degradation rate is slower than previously thought, especially in dextrose containing parenteral nutrition solutions. ${ }^{12}$ Therefore, parenteral nutrition solutions containing free glutamine can be stored at $4^{\circ} \mathrm{C}$ for at least a few days before clinical use. The study by Powell-Tuck et al (see page 82) is one of a handful of studies that has included free glutamine in parenteral nutrition solutions. Stable synthetic glutamine containing dipeptides are also available as an alternative source of glutamine. In considering how much glutamine should be added to parenteral nutrition solutions it is useful to refer to the normal dietary intake. Surprisingly, the quantities of glutamine in normal diets are not accurately known because food tables usually report glutamine and glutamate in combination (glutamine is liberated during hydrolysis of proteins and is converted to glutamate). However, it is estimated that roughly $5 \mathrm{~g}$ glutamine are ingested daily by healthy people, and a similar amount is probably delivered by enteral tube feeds. In Powell-Tuck et al's study 20 g glutamine was administered each day (3.8 g nitrogen, which is equivalent to about one third of the normal dietary nitrogen intake). Other studies have used even more glutamine (see later). Therefore, any effects of glutamine in such studies can be regarded as pharmacological. Conversely, the net inter-organ flux of glutamine in normal humans, ${ }^{3}$ which is at least $25 \mathrm{~g} /$ day (muscle, lung, adipose tissue produce glutamine, and splanchnic bed and lymphocytes utilise glutamine), approximates more closely to the daily dose of parenteral glutamine. In disease states the utilisation of glutamine often increases, with the result that there is a reduction in both the circulating glutamine concentration and the size of the free glutamine pool, which is largely located in muscle.

Powell-Tuck et al's study differs from a number of others on glutamine containing parenteral nutrition in including patients with a broad range of diagnoses, age (18-85 years), and clinical status, although those selected for parenteral nutrition by the nutrition team were generally very sick, as shown by their high hospital mortality and prolonged hospital stay. As glutamine has been implicated in having a wide range of beneficial effects (e.g. on immune function, gut barrier function, cytokine responses, and improvement in the function of a variety of tissues, including the heart) it might also prove to be beneficial to a wide range of patients. Therefore, the study set out to test the hypothesis that glutamine could be beneficial in routine clinical practice. The number of patients in the study was 168 , which was considered to be sufficiently large to detect a 10 day reduction $(-30 \%)$ in the length of hospital stay with an $80 \%$ power. No overall significant difference was found between the glutamine supplemented and control groups of patients in mortality (17 $v 24 \%$ respectively), in the incidence of infective complications (38 $v 56 \%$ of patients), or length of hospital stay ( $32 v 35$ days). It is possible that certain groups of patients benefited from glutamine supplementation, but the number of patients within these groups may not have been sufficiently large to test statistically this possibly. However, the significant reduction $(p<0.03)$ in the length of hospital stay in the surgical patients (30 $v 45$ days) is of considerable interest, especially as another recent study reported that parenteral nutrition containing a glutamine dipeptide $(0.2 \mathrm{~g}$ glutamine $/ \mathrm{kg} /$ day) reduced the length of hospital stay in patients undergoing elective colonic surgery by six days $(22$ $v 16$ days; $\mathrm{p}<0.05){ }^{4}$

The only other parenteral nutrition study that assessed the effect of glutamine on a very heterogeneous group of adult patients was that of Griffiths et $a l^{5}$ which involved critically ill patients in an intensive care unit (ICU). Eighty four patients were randomised to receive either $25 \mathrm{~g}$ glutamine/day or a mixture of non-essential amino acids (see table 6 for comparison of amino acid composition between groups in Powell-Tuck et al's study). Again, there was no significant reduction in the complication rates or mortality during their stay in the ICU or hospital, but the six month mortality, which included a post-discharge period, reached statistical significance in favour of the glutamine group $(p<0.049)$. Other clinical studies have produced variable results. In adult bone marrow transplant patients receiving cytotoxic drugs a reduction in the length of hospital stay and infective complications was found in patients receiving glutamine enriched parenteral nutrition (0.57 g glutamine/kg/day) compared with the control group that received no glutamine. ${ }^{6}$ In contrast, glutamine enriched parenteral nutrition (26 g glutamine/day $)^{7}$ ) or oral glutamine (15 $\mathrm{g} /$ day $)^{89}$ in adult patients receiving chemotherapy has been reported to produce no benefit in relieving toxicity such as oral mucositis, which often causes pain and reduces food intake. Similarly, glutamine supplemented enteral feeding has also been reported to reduce the requirement for ventilation and time to full oral feeding in premature babies weighing less than but not in those weighing more than 800 g. ${ }^{10}$ An early report in adults suggests that glutamine supplemented enteral nutrition reduces infectious complications following multiple trauma. ${ }^{11}$ 
The routine inclusion of at least some glutamine in parenteral nutrition solutions can be regarded as rational because it "humanises" the intravenous diet. The use of large pharmacological doses of glutamine may be of benefit in some specific conditions but Powell-Tuck et al's study fails to provide evidence of overall benefit in routine clinical practice.

Finally, it is appreciated that these studies are time consuming, often taking years to complete ( 20 months in the case of the Powell-Tuck study). Researchers must be persistent in adhering to their study design, remain blind to the treatment, and resist any changes in treatment that might interfere with the outcome of the study and therefore Powell-Tuck and colleagues' study, which is the largest of its kind, has been a major undertaking. Large multicentre studies perhaps provide the only practical way of rapidly assessing the possible benefits of glutamine in large subgroups of patients.

MRC Dunn Human Nutrition Unit

M ELIA

Hills Road,

Cambridge CB2 2DH, UK
1 Khan K, Hardy G, McKelroy B, et al. Stability of L-glutamine in total parenteral nutrition solutions. Clin Nutr 1991;10:193-8.

2 Khan K, Elia M. Factors affecting stability of L-glutamine in solution. Clin Nutr 1991;10:186-92.

3 Elia M. Glutamine metabolism in human adipose tissue in vivo. Clin Nutr 1993;12:50-3.

4 Morlion B, Stehle P, Wachtler P, et al. Total parenteral nutrition with glutamine dipeptide after major abdominal surgery. Ann Surg 1998;227: 302-7.

5 Griffiths RD, Jones C, Palmer TE. Six-month outcome in critically ill patients given glutamine-supplemented parenteral nutrition. Nutrition patients given glita.

6 Zeigler TR, Young LS, Benfell K, et al. Clinical and metabolic efficacy of glutamine-supplemented parenteral nutrition after bone marrow transplantation. A randomised, double-blind, controlled study. Ann Intern Med 1992;116:821-8

7 van Zaanen HCT, van der Lelie H, Timmer JG, et al. Parenteral glutamine dipeptide supplementation does not ameliorate chemotherapy-induced toxicity. Cancer 1994;74:2879-84.

8 Jebb SA, Marcus R, Wetherall A, et al. A pilot study of oral glutamine supplementation in patients receiving bone marrow transplantation. Clin Nutr 1995;14:35-41.

9 Jebb SA, Osborne RJ, Maughan TS, et al. 5-fluorouracil and folinic acid induced mucositis: no effect of oral glutamine supplementation. Br f Cancer 1994;70:732-5.

10 Lacey M, Crouch JB, Benfell K, et al. The effects of glutaminesupplemented parenteral nutrition in premature infants. FPEN f Parent Enteral Nutr 1996;20:74-80.

11 Hondijk AP, Rijnsburger ER, Jansen J, et al. Randomised trial of glutamineenriched nutrition on infectious morbidity in patients with multiple trauma. Lancet 1998;352:772-6.

\section{Sex and drugs and HCV?}

Patients infected with hepatitis $\mathrm{C}$ virus (HCV) often ask whether they might pass the virus to their sexual partners and some ask whether they might have acquired their infection through sex. Common sense would suggest that $\mathrm{HCV}$, like hepatitis B and HIV, can be transmitted through sexual contact. The issues surrounding HCV and sex can be honed into a series of focused questions. Does sexual contact carry a risk of transmitting HCV? If so, how big is the risk? Does the size of the risk vary between groups of patients? Do specific behaviours influence the size of this risk? We are close to knowing the answers to some of these questions, the answers to others are surmised but none is known with certainty.

Convincing evidence of sexual transmission requires a history of contact, the absence of other opportunities for infection, a credible temporal association, and viral genetic evidence that both partners were infected with the same virus. High quality case reports might hold the answer. ${ }^{1}$ Soon after HCV was cloned, reports of sexual transmission began to appear in the literature and it is now widely assumed that HCV can be passed through sex but few reports satisfy all criteria. In particular, it is rarely possible to exclude all other potential routes of transmission, even ones seemingly as innocuous as the shared use of a razor or toothbrush. Many reports have relied upon HCV genotyping alone without genetic sequence analysis to confirm that both partners were infected with the same virus. Both these failings are explored in a report by Zylberberg et al in this issue (see page 112).

The other questions are harder to answer and require population studies that satisfy stringent criteria of validity and applicability. The magnitude of the risk of transmitting HCV through sex should be investigated in a prospective cohort study in which a group of couples discordant for infection, assembled at an early time point in their relationship, are followed to see whether they pass the virus to their partners. Lifestyle questionnaires documenting sexual behaviour and other known risk factors should be recorded. Viral genetic evidence would be required to confirm or refute virus sharing. No such study has yet been performed and logistic and ethical considerations mean that it is unlikely ever to be performed. In its absence we must rely on data acquired from studies of less valid methodology, such as case series and case-control studies.

Reports of such studies abound, but their generalisability is poor because they have been conducted in well circumscribed but widely differing groups of subjects. Not surprisingly, their estimates of risk vary enormously from $0 \%$ to $27 \%$. Some of the clearest evidence comes from the follow up of couples where one partner was infected through a blood product. These groups include women exposed to anti-Rhesus $\mathrm{D}^{23}$ and haemophiliacs. ${ }^{45}$ The vast majority of these studies estimate the risk of sexual transmission to be between $0 \%$ and $3 \%$. However, the route and nature of the exposure, and the sex of the index partner for the anti-D mothers, differ from most patients presenting to liver clinics with HCV infection. The low rate of transmission between these couples, in which other risk factors are rare, suggests that sexual transmission is likely to be infrequent.

Why have some investigators found higher rates of transmission in other patient groups? In these studies most of the index patients' partners have been exposed to other potential routes of transmission, the predominant one being intravenous drug use (IVDU). The reports of research in specific patient groups such as those attending genito-urinary medicine clinics, IVDUs, homosexuals, and prostitutes provide valuable information but their findings are probably not generalisable to other patient groups. Some investigators have attempted to identify specific behaviours that increase the likelihood of acquiring HCV. Two extensive studies have failed to demonstrate an association between HCV infection and a wide varitety of practices thought to increase HIV transmission, including oro-anal sex, "fisting", and unprotected anal receptive sex with ejaculation. ${ }^{6}$ However, co-infection with sexually transmitted diseases, particularly herpes simplex $2,{ }^{8}$ has been specifically associated with HCV. Each of these studies provides additional data that might be useful when 
attempting to provide individualised and relevant information for patients. However, most patients attending liver clinics for advice and treatment neither correspond to these high risk patients nor do they have the limited risk of exposure to a blood product. What can we tell these patients about the likelihood that they contracted HCV through sex and their risk of transmitting the infection to their sexual partners?

Zylberberg et al report interesting and valuable data concerning the sexual transmission of HCV. They ask one clear question: in sexually active couples sharing $\mathrm{HCV}$ infection attending a routine liver clinic, how often is sexual transmission clearly not the route by which the partners have infected one another? Their study was conducted in a routine, inner city liver clinic, with a high prevalence of HIV infection, but otherwise not unlike the majority of liver clinics in Europe seeing unselected HCV infected patients. They administered a lifestyle questionnaire and performed HCV PCR and sequence analysis in consenting couples. Refusal to give consent to testing prevented them from measuring the prevalence of $\mathrm{HCV}$ infection in the partners of $\mathrm{HCV}$ infected individuals or the risk of sexual transmission of HCV. They found that sexual transmission may be mistaken for other routes of transmission.

They have searched 1640 patients to identify 24 who had HCV antibody positive partners. In three couples both partners shared identical HCV sequences and in each case other risk factors for transmission could be identified. IVDU was particularly common in their study, a finding that will be familiar to many involved in the care of $\mathrm{HCV}$ infected patients and their partners. The necessity for genetic sequence analysis to confirm that partners share the same virus is well illustrated by the investigators. No specific sexual behaviours or infections were found to be associated with $\mathrm{HCV}$ infection but the study was insufficiently powered to be informative. The authors draw attention to as yet unrecognised routes of transmission.

Sexual transmission of $\mathrm{HCV}$ is probably an infrequent occurrence. The present study emphasises that transmis- sion of HCV between partners should not be attributed to sexual contact when other routes have not been considered and explored, especially as some of these, such as IVDU, represent far greater and modifiable risks of transmission. The true extent of the risk of sexual transmission of HCV by unselected patients has still to be established but when counselling patients, appropriate emphasis should be placed on changing behaviours known to carry a high risk of transmission, such as IVDU, and life style choices known to effect adversely the course of disease, such as drinking alcohol. ${ }^{9}$

W ROSENBERG

Department of Medicine and

Division of Cell and Molecular Medicine,

Universtiy of Southampton,

811 Level D, South Block,

Southampton General Hospital,

Tremona Road,

Southampton SO16 6YD, UK

email:wmr@soton.ac.uk

1 Capelli C, Prati D, Bosoni P, et al. Sexual transmission of hepatitis C virus to a repeat blood donor. Transfusion 1997;37:436-40.

2 Sachithanandan S, Fielding JF. Low rate of HCV transmission from women infected with contaminated anti-D immunoglobulin to their family contacts. Ital f Gastroenterol Hepatol 1997;29:47-50.

3 Meisel H, Reip A, Faltus B, et al. Transmission of hepatitis C virus to children and husbands by women infected with contaminated anti-D immunoglobulin. Lancet 1995;345:1209-11.

4 Brackmann SA, Gerritzen A, Oldenburg J, et al. Search for intrafamilial transmission of hepatitis C virus in hemophilia patients. Blood 1993;81: transmission.

5 Hallam NF, Fletcher ML, Read SJ, et al. Low risk of sexual transmission of hepatitis C virus. F Med Virol 1993;40:251-3.

6 Bodsworth NJ, Cunningham P, Kaldor J, et al. Hepatitis C virus infection in a large cohort of homosexually active men: independent associations with HIV-1 infection and injecting drug use but not sexual behaviour. Genitourin Med 1996;72:118-22.

7 Buchbinder SP, Katz MH, Hessol NA, et al. Hepatitis C virus infection in sexually active homosexual men. F Infect 1994;29:263-9.

8 Shev S, Widell A, Bergstrom T, et al. Herpes simplex virus-2 may increase susceptibility of the sexual transmission of hepatitis C. Sex Transm Dis 1995;22:210-16.

9 Poynard T, Bedossa P, Opolon P. Natural history of liver fibrosis progression in patients with chronic hepatitis C. The OBSVIRC, METAVIR, CLINIVIR, and DOSVIRC groups. Lancet 1997;349:825-32.

\section{Cryoglobulinaemia in $\mathrm{HCV}$ infection: coming in from the cold}

Hepatitis $\mathrm{C}$ virus (HCV) antibodies are detected in up to $1.5 \%$ of blood donors worldwide and $\mathrm{HCV}$ is estimated to infect at least 4 million people in the USA alone. ${ }^{1} \mathrm{HCV}$ is probably the commonest cause of liver disease and hepatocellular carcinoma in the developed world and has become the leading reason for liver transplantation in many centres. $\mathrm{HCV}$ is thought to be the aetiological factor in the majority of cases of mixed cryoglobulinaemia in which $\mathrm{HCV}$ RNA is concentrated in high titre in cryoprecipitates. ${ }^{2}$ Conversely, up to $54 \%$ of patients with $\mathrm{HCV}$ infection have detectable cryoglobulins, which are associated with symptoms in roughly $25 \%$, most commonly including cutaneous manifestations and arthralgias but much less often glomerulonephritis and neurological abnormalities. ${ }^{3} \mathrm{Al}-$ though some studies have noted a correlation between the severity of liver disease and the level of cryoglobulins in serum, this has not been a consistent observation.

In this issue (see page 122), Cresta and colleagues studied 87 patients with $\mathrm{HCV}$ related liver disease prospectively. Forty three of the patients had detectable cryoglobulins, associated with symptoms in 12 (28\%). All patients were treated with 3 million units of interferon (IFN) $\alpha$ for six months, at the end of which time the biochemical, virological and immunological (disappearance of cryoglobulins) responses were $57 \%, 34 \%$, and $40 \%$, respectively. Sustained responses six months after completion of treatment were seen in $21 \%, 18 \%$ and $14 \%$ of patients, respectively, and were independent of the presence of cryoglobulins at entry. Immunological responses were associated with resolution of cutaneous and joint symptoms but neither neurological nor renal abnormalities and notably, all patients $(n=6)$ with a sustained immunological response also had a sustained virological response. Rates of initial and sustained responses tended to be higher (non-significantly) for asymptomatic patients and a long term sustained response was seen in only one patient with symptomatic cryoglobulinaemia. Factors predicting a response to IFN- $\alpha$ included male sex, genotype 2 or 3 and low viral load; however, the latter did not seem to affect response in patients with cryoglobulins. The authors concluded that the presence of cryoglobulins did not affect the response to treatment with IFN- $\alpha$ and the simultaneous clinical, immunological and virological responses provide further evidence for an aetiological role for $\mathrm{HCV}$ in mixed cryoglobulinaemia. Treatment IFN was tolerated well by 
all patients and no one had to be withdrawn from the study because of side effects.

The observed response rate in this series is comparable with previous studies, which have documented response rates in $42-73 \%$ of patients with cryoglobulinaemia treated with IFN- $\alpha$ and a disappointingly low long term response in $0-22 \%$ of patients. ${ }^{4-7}$ It is difficult to compare and reach a general conclusion from this group of studies as the clinical and immunological parameters of response are not comparable between the groups. Moreover, the treatment regimen, including dose, frequency and duration of therapy, differed in all the studies. However, some important clinical issues have been tackled by other authors. Many studies had a $10-14 \%$ dropout rate because of intolerable side effects, with one study noting a deterioration in renal and neurological symptoms while on treatment, whereas other patients required a reduction in dose. These problems were not encountered by Cresta and colleagues, perhaps because of the lower dose of IFN- $\alpha$ used. Other comparable observations were the association of genotype 2 with response to treatment, the poor response of neurological symptoms in general and the tendency (though not significant) for symptomatic patients to respond less well than asymptomatic ones. Although not a universal finding, immunological responses tend to mirror viral responses, implying that the success of IFN- $\alpha$ relies on its anti-viral action rather than effects on immune function.

Marginal improvements in the long term responses have been achieved with higher doses of IFN- $\alpha$ and a longer duration of treatment, as observed by Casato and colleagues $^{6}$ and Adinolfi and coworkers ${ }^{7}$ who treated their patients for 12 months, obtaining long term response rates of 16 and $22 \%$, respectively. It is likely that the addition of ribavirin to IFN- $\alpha$ will improve the virological response further, as already documented in patients with $\mathrm{HCV}$ associated liver disease. ${ }^{89} \mathrm{~A}$ proportion of patients in previous studies has responded when treated with IFN- $\alpha$ monotherapy for a second time; this group may now be more suitably treated with combination therapy with which long term response rates of $49 \%$ have been achieved in relapsers receiving combination therapy as opposed to only $5 \%$ with monotherapy. ${ }^{10}$

Although IFN therapy is still the most extensively used antiviral agent for chronic HCV infection, it is far from ideal and the search continues for more effective antiviral agents and combination treatment.
In the meantime, what lessons can be learnt from this and similar studies? Firstly, that the presence or absence of circulating cryoglobulins cannot be used to identify patients with more severe liver disease nor to identify those more likely to respond to IFN- $\alpha$. Secondly, that patients with cryoglobulinaemia and associated symptoms could be considered for treatment irrespective of the severity of liver disease as there is a reasonable expectation of symptomatic relief following a virological response. Resolution of the less common but more severe complications (nephrological and neurological) is less likely however. Thirdly, should we continue to look for cryoglobulinaemia? Probably not, except where symptoms or biochemical findings suggest they are clinically relevant as in most instances cryoglobulinaemia is of little clinical importance. Finally, what do we know of the mechanisms that underlie the formation of cryoglobulins and their association with $\mathrm{HCV}$ rather than - for example, hepatitis B virus infection? So far, very little.

\section{O M CROSBIE}

G J M ALEXANDER

Department of Clinical Medicine,

Cambridge University School of Clinical Medicine,

Addenbrooke's NHS Trust,

Cambridge CB2 2QQ, UK

1 Alter MJ. Epidemiology of hepatitis C. Hepatology 1997;26:625-55

2 D'Amico G. Renal involvement in hepatitis C infection: cryoglobulinaemic glomerulonephritis. Kidney Int 1998;54:650-71.

3 Wong VS, Egner W, Elsey T, et al. Incidence, character and clinical relevance of mixed cryoglobulinemia in patients with chronic hepatitis virus of mixed cryoglobulinemia in patients

4 Misiani R, Bellavita P, Fenili D, et al. Interferon alfa-2a therapy in cryoglobulinemia associated with hepatitis C virus. N Engl f Med 1994;330 globulin.

5 Casato M, Agnello V, Pucillo LP, et al. Predictors of long-term response to high-dose interferon therapy in type II cryoglobulinemia associated with hepatitis C virus infection. Blood 1997;90:3865-73.

6 Ferri C, Marzo E, Longombardo G, et al. Interferon $\alpha$ in mixed cryoglobulinemia patients: a randomised, crossover-controlled trial. Blood 1993;81:1132-6.

7 Adinolfi LE, Utili R, Zampino R, et al. Effects of long-term course of alphainterferon in patients with chronic hepatitis $\mathrm{C}$ associated to mixed cryoglobulinaemia. Eur f Gastroenterol Hepatol 1997;11:1067-72.

8 Reichard O, Norkrans G, Fryden A, et al. Randomised, double-blind, placebo-controlled trial of interferon $\alpha-2 b$ with and without ribavirin for chronic hepatitis C. Lancet 1998;351:83-7.

9 Poynard T, Marcellin P, Lee SS, et al. Randomised trial of interferon $\alpha 2 b$ plus ribavirin for 48 weeks or for 24 weeks versus interferon $\alpha 2 \mathrm{~b}$ plus placebo for 48 weeks for treatment of chronic infection with hepatitis $\mathrm{C}$ virus. Lancet 1998;352:1426-32.

10 Davis GL, Esteban-Mur R, Rustgi V, et al. Interferon alfa-2b alone or in combination with ribavirin for the treatment of relapse of chronic hepatitis C. N Engl F Med 1998;339:1493-9.
Endoscopic retrograde cholangiopancreatography (ERCP) is an advanced endoscopic procedure that requires considerable training and experience to perform effectively and safely. ${ }^{1}$ It is the most dangerous procedure regularly performed by gastrointestinal endoscopists, with morbidity and mortality of $5-10 \%$ and $0.1-1 \%$, respectively. ${ }^{2}$ In the United States, recommendations for ERCP training have evolved from ridiculously low "guesstimates" of procedure numbers required for competence ${ }^{3}$ (e.g. 25,50 ) to the current consensus - based on a large prospective study ${ }^{4}$ - that at least 180-200 diagnostic and therapeutic procedures are needed. ERCP is an integral part of the management of hepatobiliary and pancreatic (HBP) disorders, but it is only a part. Trainee gastroenterologists wishing to become experts in dealing with these disorders need to learn many clinical as well as procedural skills; it is useful to think of this as HBP training. The would be HBP specialist must work as part of a multidisciplinary team, and learn a great deal about body imaging (e.g. ultrasound, computed tomography, magnetic resonance), interventional radiology, gastrointestinal pathology and cytology, hepatology, oncology, surgery, and nutrition. Although perhaps only half of the cases seen in a specialist unit require ERCP, those intending to perform this procedure should be fully trained. There is no place for the purely "diagnostic" ERCP endoscopist: all endoscopists who perform ERCP must be able to decompress an obstructed biliary tree by sphincterotomy, stenting or nasobiliary drainage. ${ }^{5}$ The trained ERCP endoscopist's success rate for cannulating the duct of choice (common bile duct or pancreatic duct) 
should exceed $80 \%{ }^{6}$ (90-95\% in a specialist unit). Undertrained endoscopists with success rates for ERCP in the $25-50 \%$ range abound, especially in the United States. Many are self-taught or have very limited training based on weekend courses using static or animal models. ${ }^{7}$ They often fail to cannulate, or perform incomplete studies, leaving obstructed biliary or pancreatic ducts undrained. Their complication rates are high as a result of repeated instrumentation of the duodenal papilla and inept use of standard and needle-knife papillotomy. ${ }^{8}$ Their lack of experience of interpreting ERCP radiology not infrequently results in missed or erroneous diagnoses, and inappropriate use of therapeutic ERCP techniques. There are many reasons to insist on standardised ERCP training for HBP specialists: it ensures the best care for patients, minimises risk and maximises benefit and protects physicians and their employers from litigation.

Who performs the ERCP (gastroenterologists, surgeons, radiologists) is less important than the quality of their training. In this issue, Wicks et al (see page 154) recommend the development of structured ERCP training and assessment in the United Kingdom to meet the demands of the Calman (and soon to be post-Calman) era. As experienced teachers, they recognise that not all gastrointestinal trainees can or should receive ERCP training. They also appreciate the need for ERCP to be learned in an environment where all the necessary clinical, procedural and interdisciplinary resources are readily available. To be able to perform diagnostic and therapeutic ERCP with an understanding of their risks and benefits, longitudinal experience of caring for inpatients and outpatients with HBP disorders is essential. Novices who focus on acquiring the technical skills of ERCP and neglect to study the disease processes they are used to investigate and treat are a menace to their patients.

As HBP and ERCP training are inexorably linked, let us assume that HBP training includes structured experience of diagnostic and therapeutic ERCP. If we accept that HBP training has to be rationed to maximise the experience of individual trainees, then a selection process is needed. How do we choose who gets HBP training? Solid clinical and endoscopic skills are essential, as well as a clear interest in HBP disorders and their management. How can we ensure that the selection process is fair? Will trainees in Aberdeen and Aberystwyth have the same opportunities as those in London, Leeds and Liverpool? It is important to avoid a glut of would be HBP specialists, otherwise too many endoscopists will be performing too few ERCPs to maintain proficiency. HBP training should not be the reward for furious lobbying, nor for being the professor's anointed. The fairest way to choose HBP trainees would be to establish nationally agreed criteria and have the selection process overseen by one or more professional bodies or societies. The fact that physicians from a variety of disciplines perform ERCP in Britain complicates the oversight issue. Inevitably, local factors will continue to dictate how candidates are chosen for training. Selection for HBP training should not be a guarantee of accreditation in that subspecialty at the end of a predetermined period. Unfortunately, some trainees lack the necessary hand-eye coordination or cognitive skills to perform ERCP; in my experience, this is usually irremediable, regardless of how many procedures are performed. These individuals should be identified quickly and advised appropriately; it is no kindness to let them struggle on. They should be told gently but firmly that their future is not in managing HBP disorders and offered assistance to change their trajectory towards another, less endoscopically oriented area of gastroenterology. In this litigious age, the events leading to such action must be meticulously documented, to avoid possible law suits alleging prejudice or victimisation. Every trainee should keep a record of their procedures. With the availability of computerised systems for endoscopy report generation and data storage, any unit large enough to offer HBP training should be able to provide its trainees with printed reports to file in loose leaf folders. To ensure that trainees do not exaggerate their ERCP numbers, each report should be cosigned by a trainer who can to attest to the trainee's active involvement. For an ERCP to count towards training, it is essential that the trainee spend time as the sole manipulator of the instrument. Just being in the room when the ERCP is performed - namely observational experience-does not constitute training. When job applicants claim to have done a surprisingly large number of ERCPs during training, this information is best viewed with a jaundiced eye. Training numbers must be verifiable, and this should be made the responsibility of the trainer. At the end of the training period, the trainer should produce a written report detailing the trainee's experience, including specific numbers of procedures. This could be done using a standard form that would be submitted when trainees apply for senior registrar or consultant posts. Endoscopic training directors in the United States routinely issue such reports at the request of hospital authorities.

Should ERCP be performed only in specialist centres? HBP training should be conducted in specialist units with the required volume of patients and procedures and all the necessary support services. The teaching centres should teach and provide specialist services not readily available in the community, but to have them monopolise ERCP would deny many well-trained gastroenterologists, surgeons and radiologists the opportunity to apply their hard-won skills. An appropriately trained ERCP endoscopist in the community can do much useful work, and manage HBP emergencies, such as acute cholangitis, with the necessary equipment, technical support and referral centre back up. The inglorious days of "see one, do one, teach one" in gastrointestinal endoscopy are (thankfully) long gone. We should choose a few good men and women every year and train them really well to manage HBP disorders. Not everyone will agree, but this is surely the future of ERCP training.

Director, Biliary Service, Duke University Medical Centre,

J BAILLIE

Durham, North Carolina 27710, USA

1 Leung JW, Chung RS. Training in ERCP. Gastrointest Endosc 1992;38: 517-18

2 Freeman ML, Nelson DB, Sherman S, et al. Complications of endoscopic biliary sphincterotomy. N Engl f Med 1996;335:909-18.

3 Health and Public Policy Committee, American College of Physicians. Clinical competence in diagnostic endoscopic retrograde cholangiopanClinical competence in diagnostic endoscopic
creatography. Ann Intern Med 1988;108:142-4

4 Jowell PS, Baillie J, Branch MS, et al. Quantitative assessment of procedural competence. A prospective study of training in endoscopic retrograde cholangiopancreatography. Ann Intern Med 1996:125:983-9.

5 Baillie J. ERCP for all? Gastrointest Endosc 1995;42:373-6.

6 Watkins JL, Etzkorn KP, Wiley TE, et al. Assessment of technical competence during ERCP training. Gastrointest Endosc 1996;44:41 1-15.

7 Noar MD. An established porcine model for animate training in diagnostic and therapeutic ERCP. Endoscopy 1995;27:77-80.

8 Baillie J. Needle knife papillotomy revisited [editorial]. Gastrointest Endosc $1997 ; 46: 282-4$. 